\title{
Correction to: Predictive Rate-Distortion for Infinite-Order Markov Processes
}

\author{
Sarah E. Marzen ${ }^{1}$. James P. Crutchfield ${ }^{2}$ \\ Published online: 13 February 2021 \\ ○) Springer Science+Business Media, LLC, part of Springer Nature 2021
}

\section{Correction to: J Stat Phys https://doi.org/10.1007/s10955-016-1520-1}

We uncovered an error in Ref. [1] that claims the predictive rate-distortion function separates achievable from unachievable sensors. This relies on the standard rate-distortion theorem, but the latter does not apply.

Reference [2] invokes a slightly different setup-one closer to that used by Shannon himself. There, an information source is coded, sent to a channel, and decoded to uncover the original source. (To map from this to Ref. [1], simply coarse-grain the first code and channel into a single code.) Reference [2] shows that an optimal communication system-in which the code is well-matched to the channel or equivalently (Lemma 2) - can only be optimal if the distortion measure satisfies a particular constraint that corresponds, in essence, to the information bottleneck (Lemma 4i).

As such, Ref. [1] overstated the generality of the result in question. It is still true that the proposed predictive rate-distortion objective-which is very general in terms of allowed distortion measures - can often be reformulated in terms of forward-time and reverse-time causal states. However, the objective itself corresponds to an optimal communication system only when the distortion measure is the conditional entropy. And, this setup corresponds to the predictive information bottleneck.

The original article can be found online at https://doi.org/10.1007/s10955-016-1520-1.

James P. Crutchfield

chaos@ucdavis.edu

1 Department of Physics, Redwood Center for Theoretical Neuroscience, University of California at Berkeley, Berkeley, CA 94720-5800, USA

2 Complexity Sciences Center and Department of Physics, University of California at Davis, One Shields Avenue, Davis, CA 95616-5720, USA 


\section{References}

1. Marzen, S., Crutchfield, J.P.: Predictive rate-distortion for infinite-order Markov processes. J. Stat. Phys. 163(6), 1312-1338 (2014)

2. Gastpar, M., Rimoldi, B., Vetterli, M.: To code, or not to code: Lossy source-channel communication revisited. IEEE Trans. Inf. Theory 49(5), 1147-1158 (2003)

Publisher's Note Springer Nature remains neutral with regard to jurisdictional claims in published maps and institutional affiliations. 